\title{
PERFIL EPIDEMIOLÓGICO DOS PACIENTES COM PARALISIA CEREBRAL ATENDIDOS NA CLÍNICA DE FISIOTERAPIA DA UNIVERSIDADE SÃO JUDAS TADEU
}

\section{Natália Silva Carvalho dos Santos ${ }^{1}$, Larissa da Conceição Moreira', Maiara Lima Almeida', Gislene Gomes da Silva ${ }^{2, A}$, Patricia Martins Franciulli ${ }^{3}$}

'Discentes do curso de graduação em fisioterapia - Universidade São Judas Tadeu (USJT) - São Paulo - Brasil.

${ }^{2}$ Preceptora do estágio em Saúde Coletiva - Universidade São Judas Tadeu (USJT) - São Paulo - Brasil. Discente do Programa de mestrado da FMSCMSP

[2Docente do curso de fisioterapia da Universidade São Judas Tadeu (USJT) - São Paulo - Brasil

\begin{abstract}
RESUMO
Introdução: A paralisia cerebral (PC) é uma doença causada por lesōes ao encéfalo em desenvolvimento, sendo a principal fonte de deficiência física em crianças, podendo acarretar déficits nas funções neuromusculares que afetam diretamente a qualidade de vida. Objetivo: Avaliar o perfil epidemiológico dos pacientes com PC atendidos na clínica de fisioterapia da Universidade São Judas Tadeu (CFUSJT). Método: Trata-se de uma pesquisa quantitativa do tipo exploratório-descritiva, utilizando-se de estudo retrospectivo. Os dados foram colhidos dos prontuários de pacientes atendidos na CFUSJT. Critérios de inclusão: Prontuários físicos de pacientes atendidos no setor de fisioterapia neurofuncional em pediatria da CFUSJT, no periodo de fevereiro de 2010 a dezembro de 2020. Critérios de exclusão: Prontuários físicos de pacientes sem confirmação diagnóstica de PC após os dois anos de idade, além dos com mudança de diagnóstico após investigação do atraso do desenvolvimento neuropsicomotor (ADNPM). Análise dos dados: Os dados foram tabulados e foi feita a análise estatistica descritiva e percentual de cada item. Resultados: A prevalência da PC è maior no sexo masculino, acometendo em sua maioria crianças que nasceram prematuras e por via de parto normal. Aidade média destes é de $\pm 7,60$ anos, tendo como o diagnóstico fisioterapêutico predominante a tetraplegia e a diplegia espástica. As intercorrências gestacionais mais prevalentes foram a eclampsia, parto de emergência e infecção urinária; e as intercorrências com as crianças foram convulsão e anóxia, hipóxia e problemas cardiacos. Conclusão: O perfil dos pacientes atendidos na CFUSJT refere-se sexo masculino, idade média de 7,6 anos, com deficiências motoras, cognitivas e visuais, moradores da região da zona leste da cidade de São Paulo e referenciados a instituição através do SUS
\end{abstract}

Palavras chaves: Paralisia Cerebral; Fisioterapia; Prontuários

\section{ABSTRACT}

Background: Cerebral palsy (CP) is a disease caused by lesions to the developing brain, being the main source of physical disability in children, which can lead to deficits in neuromuscular functions that directly affect the quality of life. Objective: To evaluate the epidemiological profile of patients with CP treated at the physiotherapy clinic of the São Judas Tadeu university (CFUSJT). Method: This

^Autor Correspondente: Gislene Gomes da Silva - E-mail: gisgsilva@gmail.com - ORCID: https://orcid.org/0000-0002-3980-7464

DOI: https://doi.org/10.46675/rbcbm.vai1.41 - Artigo recebido em: 11 de junho de 2021; aceito em 25 de junho 2021 ; publicado 20 de Julho de 2021 na Revista Brasileira de Ciências Biomédicas, disponivel online em uww.rbcbm.com.br. Todos os autores contribuiram igualmente com o artigo. Os autores declaram não haver conflito de interesse Este è um artigo de acesso aberto sob a licença CC - BY: http://creativecommons.org/licenses/by/4.0 
is an exploratory-descriptive quantitative research, using a retrospective study. Data were collected from the medical records of patients treated at the CFUSJT. Inclusion criteria: physical medical records of new patients seen at pediatrics neurofunctional physiotherapy department in CFUSJT, from February 2010 to December 2020. Exclusion criteria: physical medical records of patients without diagnostic confirmation PC after two years of age, in addition to those with a change in diagnosis after investigation of neuropsychomotor development delay (ADNPM). Data analysis: Data were tabulated and descriptive statistical analysis and percentage of each item was performed. Results: The prevalence of $\mathrm{CP}$ is higher in males, affecting children who were born prematurely and through normal birth. The mean age of patients is \pm 7.60 years, with quadriplegia and spastic diplegia as the most common physiotherapeutic diagnosis. The most prevalent gestational complications were eclampsia, emergency delivery and urinary infection; and complications with children were seizure and anoxia, hypoxia and heart problems. Conclusion: the profile of patients admitted at CFUSJT is comprised of males, with an average median age of 7.6 years, having motor, cognitive and visual impairments, residents of the east area of the city of São Paulo, and referenced to the institution through the SUS.

Keywords: Cerebral palsy, Physiotherapy, Medical Records.

\section{INTRODUÇÃO}

A paralisia cerebral (PC) foi descrita pela primeira vez em 1843 por William John Little, ortopedista inglês, que estudou 47 crianças com espasticidade que possuíam apresentação pélvica, prematuridade, dificuldade no trabalho de parto, demora em chorar e respirar ao nascer, convulsões e coma nas primeiras horas de vida [1].

A etiologia da PC é multifatorial e tem como causa lesões ao encéfalo em desenvolvimento, sendo a principal fonte de deficiência física em crianças [2], apesar de ser uma doença não progressiva, pode gerar impacto diretamente na condição geral do indivíduo ao longo dos anos devido ao amadurecimento do SNC [3]. Dentre esses fatores causais podemos citar as intercorrências pré-natais como as causas genéticas, malformações cerebrais, infecções congênitas, exposição a agentes tóxicos como álcool e tabaco, alterações nutricionais, hipóxia intrauterina, alterações perinatais como a prematuridade e anóxia [4] e fatores pósnatais que ocorrem em cerca de $10-18 \%$ dos casos, como sepse, meningite [3], síndromes convulsivas, causas metabólicas, afogamento, tumores e encefalopatias [5].

Esses indivíduos comumente apresentam sequelas que levam ao aumento do tônus muscular, fraqueza, contraturas, déficits cognitivos, de comunicação e equilíbrio, além de restrição da amplitude de movimento que, com o passar da idade, pode se agravar e apresentar alterações na marcha e da função motora [6]. Essas desordens podem ocorrer em qualquer momento da vida do indivíduo até os 2 anos de idade [7]. A expectativa de vida de pessoas com PC varia de acordo com o tipo de deficiência apresentada, sendo que os portadores dos tipos mais graves vêm a óbito antes de chegar à fase adulta. Quase $85 \%$ dos que passam dos 20 anos sobrevivem até os 50 anos [8].

Apatologia pode ser classificada de acordo com a característica clínica mais dominante: espástico, discinético (atetóide) e atáxico. A PC espástica caracteriza-se pela presença de tônus elevado e é ocasionada por uma lesão no sistema piramidal. A espasticidade é predominante em crianças nascidas pré-termo e ocorro por lesão extrapiramidal, enquanto a forma discinética e atáxica são frequentes nas crianças nascidas a termo, tratando-se de uma lesão encefálica [1].

Para a avaliação, de forma padronizada, dos níveis de mobilidade funcional em crianças com PC é utilizado o Sistema de Classificação da Função Motora Grossa (GMFCS), o qual é baseado no movimento iniciado voluntariamente, com ênfase no sentar, transferências e mobilidade [1]. Esse sistema é dividido em 5 níveis: NÍVEL I - Anda sem limitações; NÍVEL II - Anda com limitações; NÍVEL III - Anda utilizando um dispositivo manual de mobilidade; NÍVEL IV - Auto mobilidade com limitações (pode utilizar mobilidade motorizada); NÍVEL V - Transportado em uma cadeira de rodas manual [9].

No Brasil, a incidência da PC é de 7 a cada 1000 crianças nascidas vivas, o que acarreta 30.000 a 40.000 crianças com paralisia cerebral por ano [10]. Dado esse que pouco diferiu na última década na prevalência global da paralisia cerebral, sendo de aproximadamente 2,1 em cada 1.000 nascidos em países desenvolvidos, correspondendo a $74 \%$ dos casos em todo o mundo [11].

Nos dias atuais sabemos que a porta primária para o Sistema Único de Saúde (SUS) é a Unidade Básica de Saúde (UBS) que está relacionada a atividades ambulatoriais e de assistência à população, em que os indivíduos são referenciados de acordo com a sua região [12]. É de extrema importância que tenhamos ciência do referenciamento destes pacientes, uma vez que a vigilância em saúde pode auxiliar na necessidade de reorganização dos serviços, identificando qual a demanda de atenção que aquela população necessita, visando um planejamento local para construção de um sistema de vigilância de doenças e agravos [13].

O estudo epidemiológico visa a contribuição na identificação do perfil dos pacientes, permitindo um olhar crítico que pode utilizar de novas estratégias de ação e recursos que envolvam a proteção, promoção e recuperação da saúde voltada à população estudada [14]. Para isso, é necessário que seja feito 
um levantamento e análise de dados através de prontuários, sendo esse um documento valioso para o paciente, para equipe e para instituição de saúde [15], uma vez que o preenchimento adequado dos mesmos se torna um indicador indireto, mas relevante voltado a compressão do quadro clinico do paciente [16], contendo informações cadastrais básicas, como nome completo, data de nascimento, nome da mãe, sexo, naturalidade, endereço, anamnese, sinais vitais, exames físicos, exames complementares, hipótese diagnóstica, diagnóstico definitivos, tratamento efetuado, evolução diária, discriminação de todos os procedimentos realizados e identificação dos profissionais responsáveis [17]. Para traçar o perfil sociodemográfico de uma determinada população esses dados são imprescindíveis para a fisioterapia, podendo identificar a necessidade de cuidados fisioterapêuticos e qual o nível de atenção à saúde que esse paciente necessita [14].

\section{METODOLOGIA}

Trata-se de uma pesquisa quantitativa do tipo exploratóriadescritiva. Foi realizado um estudo retrospectivo, através da coleta de dados de prontuários físicos dos pacientes com paralisia cerebral $(\mathrm{PC})$ atendidos na clínica de fisioterapia da Universidade São Judas Tadeu (USJT) no período de 2010 a 2020, após aprovação do Comitê de Ética em Pesquisa da Instituição (CEP/ USJT), com o número do parecer 4.475.927.

A busca nos prontuários foi realizada por um grupo de pesquisadores treinados na execução do mapeamento cruzado. Foram inclusos prontuários físicos de pacientes atendidos no setor de fisioterapia neurofuncional em pediatria da CFUSJT, no período de fevereiro de 2010 a dezembro de 2020 . Houve a exclusão de prontuários físicos de pacientes sem confirmação diagnóstica de PC após os dois anos de idade, além dos com mudança de diagnóstico após investigação do atraso do desenvolvimento neuropsicomotor (ADNPM).

A amostra foi composta por 87 prontuários de pacientes com diagnóstico de paralisia cerebral de 0 a 18 anos de idade de ambos os gêneros. Posteriormente os prontuários foram avaliados minuciosamente, sendo tabulados e submetidos a análise estatística.

\section{RESULTADOS}

Após a coleta, todos os dados obtidos foram registrados em planilhas do Microsoft Office Excel para análise quantitativa e descritiva. Os dados foram descritos como média e distribuição de frequência em valores absolutos e percentuais.

Houve prevalência no sexo masculino $(59,78 \%)$ quando comparado ao sexo feminino $(40,22 \%)$ (Tabela 1), sendo utilizada uma estratificação em faixas etárias, em que a idade média desses pacientes corresponde a 7,60 anos, sendo $\leq 2$ anos (2,30\%), entre 2-4 anos (27,59\%), 5-8 anos (33,33\%), 9-12 anos (20,69\%), 13$15 \operatorname{anos}(11.49 \%), \geq 18$ anos $(2,30 \%)$ e não informaram $(2,30 \%)$ (Tabela 1). Relacionado aos tipos de paralisia cerebral (PC) e alterações anatomopatológicas foram identificados: tetraplégico espástico $(37,93 \%)$, diplégico espástico $(37,93 \%)$, hemiplégico espástico $(9,20 \%)$ e paraplégico espástico $(1,15 \%)$ e não informaram (13,79\%), conforme apresentado na Tabela 1.

Dos prontuários analisados, $29,88 \%(n=26)$ dos pacientes apresentaram deficiência cognitiva, $62,06 \%(n=54)$ deficiência motora e $21,84 \%(n=19)$ apresentavam deficiência visual, sendo que a maioria apresentava mais de uma deficiência. Os dados estão apresentados na Tabela 2.

Foram identificadas intercorrências que podem ter desencadeado a PC nestes pacientes, sendo um destes fatores o acometimento a mãe no período gestacional, destacando-se eclâmpsia $(27,04 \%)$, parto de emergência $(10,82 \%)$, infecção urinária $(8,12 \%)$ e parto demorado $(5,12 \%)$. Outros acontecimentos importantes identificados foram as intercorrências com a criança, destacando-se convulsões e anóxia (17,04\%), hipóxia (15,93\%) e problemas cardíacos $(14,80 \%)$.

Quanto ao sistema de saúde utilizado, $51 \%$ são usuários do Sistema Único de Saúde (SUS), $2 \%$ da rede privada e $47 \%$ não informaram. Através deste acompanhamento, foram referenciados a CFUSJT pelo SUS $(28,89 \%)$, indicação $(13,79 \%)$ e não informado $(56,32 \%)$.

Em sua grande maioria, os pacientes já realizaram atendimentos fisioterapêuticos anteriores em outros locais, como AACD (39,08\%), Centros Universitários (4,60\%), Home Care (3,45\%), Hospital das Clínicas (HC) $(2,30 \%)$, Particular (1,15\%) e não realizaram/não informaram $(49,43 \%)$, como representado na Tabela 3.

A Tabela 4 apresenta os dados relacionados às variáveis clínicas encontradas. A idade média gestacional corresponde a 33 semanas, sendo que $63,22 \%$ das mães realizaram pré-natal, apenas $2,30 \%$ não realizaram e $34,48 \%$ não informaram. Sobre a via de parto dos mesmos, $39,08 \%$ destes nasceram de parto normal, $37,93 \%$ de parto cesárea e $23 \%$ não informaram. Destes pacientes, $51,72 \%$ eram pré-termos ( $\leq 37$ semanas), $22,99 \%$ atermos (37-42 semanas) e 2,30\% pós termo ( $\geq 42$ semanas) e $22,99 \%$ não informaram. Esses pacientes apresentavam variações de peso ao nascimento, tendo baixo peso $31,03 \%$, peso adequado $24,14 \%$ e $44,83 \%$ não informado no ato da avaliação.

A partir do perfil sociodemográfico dos pacientes atendidos na clínica de fisioterapia da Universidade São Judas Tadeu (CFUSJT), foi possível localizar além dos dados mencionados anteriormente a região e UBS de acompanhamento, tendo maior prevalência a zona leste de São Paulo (70,27\%), como representado na Tabela 5.

\section{DISCUSSÃo}

O prontuário do paciente é um suporte informacional que deve ser organizado e de fácil acesso aos seus conteúdos, sendo protegido e amparado com legislações nacionais. O prontuário do paciente é fonte de dados e informações para o esboço de políticas públicas [18]. Com a análise dos prontuários, de acordo com o roteiro de 17 itens, foi possível construir o perfil epidemiológico dos pacientes com PC da CFUSJT. 
Tabela 1 - Refere-se ao sexo, idade e tipo de PC dos 87 pacientes atendidos na CFUSJT no período de 2010 - 2020

\begin{tabular}{lcc}
\hline Sexo & Frequência absoluta (f) & Frequência relativa (\%) \\
\hline Masculino & 52 & $59,78 \%$ \\
Feminino & 35 & $40,22 \%$ \\
Idade & & \\
$\leq 2$ anos & 2 & $2,30 \%$ \\
$2-4$ anos & 24 & $27,59 \%$ \\
$5-8$ anos & 29 & $33,33 \%$ \\
$9-12$ anos & 18 & $20,69 \%$ \\
$13-15$ anos & 10 & $11,49 \%$ \\
$\leq 18$ anos & 2 & $2,30 \%$ \\
Não informaram & 2 & $2,30 \%$ \\
Tipo de paralisia cerebral & & \\
Tetraparesia espástica & 33 & $37,93 \%$ \\
Diparesia espástica & 33 & $37,93 \%$ \\
Hemiparesia espástica & 8 & $9,20 \%$ \\
Paraparesia espástica & 1 & $1,15 \%$ \\
Não informaram & 12 & $13,79 \%$ \\
\hline \multicolumn{1}{r}{ Total } & $\mathbf{8 7}$ & $\mathbf{1 0 0} \%$
\end{tabular}

Tabela 2 - Deficiência cognitiva, motora e visual dos pacientes com PC atendidos na CFUSJT.

\begin{tabular}{lcc}
\hline Deficiência motora & Frequência absoluta (f) & Frequência relativa (\%) \\
\hline Sim & 54 & $62,06 \%$ \\
Não & 13 & $14,95 \%$ \\
Não informaram & 20 & $22,99 \%$ \\
Deficiência cognitiva & & \\
Sim & 26 & $29,88 \%$ \\
Não & 17 & $19,54 \%$ \\
Não informaram & 44 & $50,58 \%$ \\
Deficiência visual & & \\
Sim & 19 & $21,84 \%$ \\
Não & 22 & $25,28 \%$ \\
Não informaram & 46 & $52,88 \%$ \\
\hline Total & $\mathbf{8 7}$ & $\mathbf{1 0 0} \%$
\end{tabular}

Tabela 3 - Tratamentos fisioterapêuticos realizados anteriormente.

Tratamento fisioterapêutico Frequência absoluta (f) Frequência relativa (\%)

\begin{tabular}{|c|c|c|}
\hline AACD & 34 & $39,08 \%$ \\
\hline Centros universitários & 4 & $4,60 \%$ \\
\hline HomeCare & 3 & $3,45 \%$ \\
\hline $\mathrm{HC}$ & 2 & $2,30 \%$ \\
\hline Particular & 1 & $1,45 \%$ \\
\hline Não informaram & 43 & $49,43 \%$ \\
\hline Total & 87 & $100 \%$ \\
\hline
\end{tabular}


Tabela 4 - Comparação das variáveis clínicas dos 87 pacientes no período pré-natal e pós natal.

\begin{tabular}{lcc}
\hline Pré natal & Frequência absoluta (f) & Frequência relativa (\%) \\
\hline Sim & 55 & $63,22 \%$ \\
Não & 2 & $2,30 \%$ \\
Não informaram & 30 & $34,48 \%$ \\
Via de parto & & \\
Cesária & 33 & $37,93 \%$ \\
Normal & 34 & $39,08 \%$ \\
Não informaram & 20 & $22,99 \%$ \\
Idade gestacional & & \\
Pré termo & 45 & $51,72 \%$ \\
A termo & 20 & $22,99 \%$ \\
Pós termo & 2 & $2,30 \%$ \\
Não informaram & 20 & $22,99 \%$ \\
Peso ao nascer & & \\
$\leq 2.500 \mathrm{~g}$ & 27 & $31,03 \%$ \\
$2.500 \mathrm{~g}$ e $\leq 4.000 \mathrm{~g}$ & 21 & $24,14 \%$ \\
Não informaram & 39 & $44,83 \%$ \\
\hline \multicolumn{1}{c}{ Total } & $\mathbf{8 7}$ & $\mathbf{1 0 0} \%$
\end{tabular}

Tabela 5 - Prevalência da região de moradia e UBS dos pacientes com PC atendidos na CFUSJT.

\begin{tabular}{lcc}
\hline Zona & Frequência absoluta (f) & Frequência relativa (\%) \\
\hline Leste & 26 & $70,27 \%$ \\
Sul & 3 & $8,10 \%$ \\
Norte & 3 & $8,10 \%$ \\
Centro & 2 & $5,40 \%$ \\
Oeste & 1 & $2,70 \%$ \\
Outros & 2 & $5,40 \%$ \\
\hline Toltal & $\mathbf{3 7}$ & $\mathbf{1 0 0} \%$
\end{tabular}

No aspecto sociodemográfico do presente estudo, notou-se o predomínio de crianças do sexo masculino, dado que também foi encontrado em estudos brasileiros $[4,19,20,21,22]$ e internacionais [23,24]. O estudo de Cavalcante et al. (2017) [25] não corrobora com tais achados em que a quantidade de pacientes do sexo feminino e masculino se mantiveram iguais.

A idade média dos pacientes atendidos foi de 7,60 anos, semelhante ao estudo Khandaker et al. (2019) [24] em que a média de idade foi 7,70 anos. Foi encontrado o maior número de atendimentos realizados em pacientes com faixa etária de 5 a 8 anos, seguido de crianças de 2 a 4 anos; dado este que corrobora com pesquisas analisadas $[4,19,20,24,25]$ em que a idade dominante se manteve entre 4 e 9 anos.

A respeito da classificação clínica e anatômica, pode ser observado predomínio de crianças com tetraplegia e diplegia espástica, corroborando os dados de Toledo et al. (2015) [19]. Estudos $[20,22,23]$ demostram que a tetraplegia foi encontrada em maior escala, seguido da diplegia. Já os achados de Binha et al. (2018) [4] corrobora que a diplegia espástica se mantém em predominância e refuta a tetraplegia espástica. Khandaker et al. (2019) [24] não corrobora com a classificação descrita neste estudo, mantendo-se em maior escala a hemiplegia/monoplegia.

Ao analisar as deficiências adquiridas em decorrência a PC, foi observado que a maioria destes apresentavam deficiências motoras, seguida das cognitivas e visuais respectivamente. Estudos $[20,23,24]$ mostram dados semelhantes ao levantamento do presente estudo, tendo as deficiências cognitivas seguidas de visuais. Os estudos citados anteriormente avaliaram as deficiências motoras quanto ao GMFCS e Fidan et al. (2014) [23] observou a presença de disfunções orais, desequilíbrios musculares e deformidades. Todavia, no presente estudo tal dado não foi coletado por não ser especificado nos prontuários da CFUSJT.

Relacionado às intercorrências gestacionais, pôde ser 
observada uma alta taxa de mães que apresentaram eclampsia, parto de emergência e infecção urinária. Dado semelhante ao estudo de Santos et al. (2019) [21] em que as complicações gestacionais mais recorrentes foram infecção urinária e eclampsia. Em outras pesquisas $[19,20]$ as causas mais comuns foram: ameaça de aborto espontâneo, sangramento vaginal e malformações, infecções congênitas, respectivamente

A respeito das intercorrências com a criança, os fatores peri e pós-natais mais comuns foram anóxia e convulsão, hipóxia e problemas cardíacos (parada cardiorrespiratória, bradicardia e sopro). Três estudos $[19,20,25]$ também observaram a presença de convulsões, hipóxia e anóxia, respectivamente. Outras intercorrências como encefalopatia e infecções também são reportadas na literatura $[21,24]$.

No presente estudo em sua maioria as mães realizaram acompanhamento pré-natal, sendo o SUS seu referenciamento primário. Ao analisar o estudo Santos et al. (2019) [21] foi observado que todas as mães realizaram acompanhamento; já no estudo Khandaker et al (2019) [24] menos da metade teve assistência médica gestacional.

$\mathrm{Na}$ análise da via de parto foi observado em maior escala o parto normal, refutando dois estudos $[4,21]$ em que a via de parto mais comum foi a cesárea. A idade gestacional prevalente correspondeu ao parto pré-termo $[4,26]$ e não corroborando com Santos et al. (2019) [21] em que a maioria nasceu de parto pós termo. Esses pacientes apresentaram baixo peso ao nascer segundo os achados de Binha et al. (2018) [4].

Dentre as limitações encontradas no presente estudo, podemos citar o preenchimento incompleto e inespecífico dos prontuários. Portanto, sugerimos que haja um olhar criterioso para o preenchimento deste documento e alterações na ficha de avaliação voltada as deficiências motoras, cognitivas e visuais e a UBS de acompanhamento, permitindo uma investigação minuciosa a respeito do perfil sociodemográfico destes pacientes.

\section{CONCLUSÃO}

Com os dados analisados nesta pesquisa, foi possível identificar o perfil epidemiológico predominante dos pacientes com PC atendidos na CFUSJT nos últimos dez anos: sexo masculino, idade média de 7,6 anos, com deficiências motoras, cognitivas e visuais, moradores da região da zona leste da cidade de São Paulo e referenciados a instituição através do SUS

\section{REFERÊNCIAS}

[1] Ministério da Saúde. Diretrizes de Atenção à Pessoa com Paralisia Cerebral. Secretaria de Atenção à Saúde Departamento de Ações Programáticas Estratégicas, Brasília - DF, 2014; v. 1, p. 71.

[2] Petersen R.; Procter C.; Donald KA.; Clinical Child Neurology, Springer International Publishing; Assessment and Management of the Child with Cerebral Palsy. Cham, Suiça. 2020; pp. 175-203. DOI: 10.1007/978-3-319-43153-6

[3] Mlodawski J.; Mlodawska M.; Pazera G.; Michalski W.;
DOmanski T.; Dolecka-Slusarczyk M.; et al. Cerebral palsy and obstetric-neonatological interventions. Ginekologia Polska Via Médica, [S. I.]. 2019; v. 90, n. 12, p. 722-727. DOI: 10.5603 / GP.2019.0124

[4] Binha AMP.; Maciel SC.; Bezerra CCA. Perfil epidemiológico dos pacientes com paralisia cerebral atendidos na AACD - São Paulo. Acta Fisiatrica, [S. I.]. 2018; v. 25 , n. 1. Artigo Original, p. 2-5. DOI: 10.11606/issn.2317-0190. v25i1a158818

[5] Pereira GN.; Costa CS.; Silva KCC. Os benefícios da atuação do fisioterapeuta em doenças perinatais. Scire Salutis, [S. I.]. 2018 ; v. 8 , n. 2, p. 115-122. DOI: 10.6008/CBPC22369600.2018.002.0012.

[6] Rasmussen HM.; Pedersen NW.; Overgaard S.; Hansen LK.; Dunkhase-Heinl U.; Petkov Y.; et al. Gait analysis for individually tailored interdisciplinary interventions in children with cerebral palsy: a randomized controlled trial. Developmental Medicine \& Child Neurology. Dev Med Child Neurol. 2019; p. 1189-1195. DOI:10.1111/dmcn.14178

[7] Amankwah N.; Oskoui M.; Garner R.; Bancej C.; G. Manuel D.; Wall R.; et al. Original quantitative research - Cerebral palsy in Canada, 2011-2031: results of a microsimulation modelling study of epidemiological and cost impacts. Health promotion and chronic disease prevention in Canada: research, policy and practice, [S. I.]. 2020; v. 40, p. 25-37. DOI: 10.24095/hpcdp.40.2.01

[8] Melo MAG.; Quinto RC.; Souza RB. Avaliação do perfil epidemiológico de pacientes com paralisia cerebral atendidos na apae do município de Sobral-CE e análise cienciométrica sobre o assunto na literatura. Essentia-Revista de Cultura, Ciência e Tecnologia da UVA, [S. I.]. 2015; v. 16, n. 2, p. 1-6.

[9] Silva DBR.; Pfeifer LI.; Funayama CAR. GMFCS - E \& R Sistema de Classificação da Função Motora Grossa Ampliado e Revisto. Dev Med Child Neurol [S. I.]. 2010; p. 214-223.

[10] Silva GG da, Romão J, Andrade EG da S. Paralisia Cerebral e o impacto do diagnóstico para a família. Rev Inic Cient Ext. 2019; 2(1):4-10.

[11] Liang X.; Tan Z.; Cao J.; Wang J.; Liu Q.; Chen T.; Effectiveness of exercise interventions for children with cerebral palsy: A systematic review and meta-analysis of randomized controlled trials. J Rehabil Med, [S. I.]. 2021; v. 53, p. 18-22. DOI 10.2340/16501977-2772.

[12] Narcizio MHS, Ávilla AL, dos Santos BPS, Rodrigues LSA, Arantes DSS, Carrijo JR. Perfil epidemiológico e social da população atendida em uma unidade básica de saúde em Cuiabá. Rev.G\&amp;S. 2013; v. 4, n. 2, p.2129-2138.

[13] Faria LS.; Bertolozzi MR. A vigilância na Atenção Básica à Saúde: perspectivas para o alcance da Vigilância à Saúde. Rev Esc Enferm USP, São Paulo. 2010; v. 4, n. 3, 44(3):789-795. DOI: 10.1590/S0080-62342010000300034.

[14] Nunes EM.; Frias RS. Perfil Sociodemográfico, Epidemiológico e do Atendimento Fisioterápico dos Pacientes de uma Clínica Escola de Fisioterapia que Funciona no Espaço de uma Unidade Básica de Saúde Particular de Foz do Iguaçu, PR. Plêiade, [S. I.]. 2017; v. 11 n. 22 p. 46-55. 
[15] Françolin L.; Brito MFP.; Gabriel CS.; Monteiro TM.; Bernardes A.; A qualidade dos registros de enfermagem em prontuários de pacientes hospitalizados. Rev. Enferm. UERJ, Rio de Janeiro. 2011; v. 20, n. 1, p. 79-83.

[16] Batista FC.; Barbosa MGV.; Garnelo L. Fabiane Corrêa et al. Avaliação da qualidade de registro em prontuário de pacientes de fisioterapia em um hospital de Manaus - AM Manual-y-Posturologia. 2013; 11(53): p. 333-340; v. 11, n. 53. p. 21

[17] Carvalho KR. O prontuário como fonte de informação. Ministério da Saúde, Porto Alegre. 2016; p. 9-13.

[18] Galvão MCB. Prontuário do paciente. Informação \& Sociedade, [S. I.]. 2012; v. 22, n. 2, p. 173-174.

[19] Toledo CAW.; Pereira $\mathrm{CHCN}_{\text {.; }}$ Vinhaes MM; Lopes MIR.; Nogueira MARJ. Perfil epidemiológico de crianças diagnosticadas com paralisia cerebral atendidas no Centro de Reabilitação Lucy Montoro de São José dos Campos. Acta Fisiatrica, [S. I.]. 2015; v. 22, n. 3, p. 118-122. DOI: 10.5935/01047795.20150023

[20] Vieira JLR.; Chagas JRLP. Perfil funcional e sóciodemográfico dos pacientes com paralisia cerebral em reabilitação num centro de referência em salvador. Revista Brasileira de neurologia e psiquiatria, [S. I.]. 2017; v. 21, n. 2, p. 100-108

[21] Santos RA.; Silva VR.; Santos JP.; Siqueira AN. Perfil epidemiológico e assistência à saúde de crianças e adolescentes com paralisia cerebral em um municipio do ES. Resid Pediat, [S. I.]. 2019; v. 9, n. 3, p. 252-260. DOI: 10.25060/ residpediatr.

[22] Oliveira PC.; Fantato WG.; Priedols AMA.; Spressão DRMS.; Audi M. Perfil das crianças com paralisia cerebral atendidas na clínica escola de fisioterapia da universidade de marilia. Brazilian Journal of Development, [S. I.]. 2018; v. 7, n. 3, p. 21465-21472. DOI: $10.34117 /$ bjdv7n3-046.

[23] Fidan F.; Baysal O. Epidemiologic Characteristics of Patients with Cerebral Palsy. Open Journal of Therapy and Rehabilitation, [S. I.]. 2014; v. 2, n. 3, p. 126-132. DOI: 10.4236/ ojtr.2014.23018.

[24] Khandaker G.; Muhit M.; Karin T.; Smithers-Sheedy H.; Novak I.; Jones C.; et al. Epidemiology of cerebral palsy in Bangladesh: a population-based surveillance study. Developmental Medicine \& Child Neurology, [S. I.]. 2019; v. 61, n. 5, p. 601-609. DOI: 10.1111/dmcn.14013.

[25] Cavalcante VMV.; Martins MC.; Oriá MOB.; Ximenes LB.; Frota LB.; Carvalho ZMF. Perfil epidemiológico das crianças com paralisia cerebral em atendimento ambulatorial. Revista de Enfermagem UERJ, [s. I.]. 2017; p. 1-7, 20. DOI: 10.12957/ reuerj.2017.8780

[26] Sadowska M.; Sarecka-Hujar B.; Kopyta I. Cerebral Palsy: Current Opinions on Definition, Epidemiology, Risk Factors, Classification and Treatment Options. Neuropsychiatric Disease and Treatment, [S. I.]. 2020; v. 16, p. 1505. DOI: 10.2147/ NDT.S235165 